\title{
Emergent symmetries and coexisting orders in Dirac fermion systems
}

\author{
Emilio Torres $\odot,{ }^{1}$ Lukas Weber $\odot,{ }^{2}$ Lukas Janssen $\odot,{ }^{3}$ Stefan Wessel $\odot,{ }^{2}$ and Michael M. Scherer ${ }^{1}$ \\ ${ }^{1}$ Institut für Theoretische Physik, Universität zu Köln, 50937 Cologne, Germany \\ ${ }^{2}$ Institut für Theoretische Festkörperphysik, JARA-FIT and JARA-HPC, RWTH Aachen University, 52056 Aachen, Germany \\ ${ }^{3}$ Institut für Theoretische Physik and Würzburg-Dresden Cluster of Excellence ct.qmat, Technische Universität Dresden, \\ 01062 Dresden, Germany
}

(Received 12 November 2019; revised manuscript received 10 February 2020; accepted 13 March 2020; published 8 April 2020; corrected 8 October 2020)

\begin{abstract}
We consider interacting (2+1)-dimensional Dirac fermions with competing symmetry-breaking electronic instabilities, as described by relativistic quantum field theories of the Gross-Neveu-Yukawa flavor with anticommuting mass terms. We demonstrate, using a combination of nonperturbative field-theoretical analysis and an adapted quantum Monte Carlo approach, that such systems exhibit a strong-coupling quantum multicritical fixed point with an emerging enhanced symmetry. Moreover, an extended phase coexistence regime expands out from this high-symmetry point. Our results disagree with recent results on the presence of a deconfined quantum criticality in (2+1)-dimensional Dirac fermions for the particular case of $O(3)$ Néel and $Z_{2}$ Kekulé symmetry-breaking instabilities on the graphene lattice. The robustness of these phenomena with respect to the microscopic symmetries furthermore demonstrates their relevance for a wide range of Dirac materials of current interest, from both theory and ongoing experiments.
\end{abstract}

DOI: 10.1103/PhysRevResearch.2.022005

Recent advances in the fabrication of two-dimensional electron systems, such as in twisted bilayer graphene, have exhibited the emergence of strongly correlated states [1,2], which are reminiscent of other material classes, e.g., the transition-metal oxides, where the interplay of competing electronic instabilities leads to rich phase diagrams [3,4]. In particular, these findings have renewed interest in the symmetry-broken phases of correlated Dirac fermions, due to the evidence of gap openings near charge neutrality [5-9]. Interacting Dirac fermion systems are also of fundamental interest from a general field-theoretical perspective, and are thus in focus of intensive recent research [10-15]. Based on these developments, Dirac fermion systems were argued to host markedly intriguing phenomena, such as the emergence of enhanced symmetry lines or the stabilization of quantum criticality beyond the realms of the traditional LandauGinzburg-Wilson (LGW) theory of critical phenomena [16]. For example, it was argued that Dirac fermions on graphene's honeycomb lattice support deconfined quantum critical points (DQCPs) [17] via the condensation of topological defects or anticommuting mass terms $[18,19]$. Moreover, recent theoretical calculations suggest that emergent $O(N)$ symmetries are a prevalent feature of deconfined quantum phase transitions [19-22] and beyond [23-32]. This recent progress raises two important, fundamental issues that are intimately related: (i) the stability of enhanced symmetry states in Dirac materials

Published by the American Physical Society under the terms of the Creative Commons Attribution 4.0 International license. Further distribution of this work must maintain attribution to the author(s) and the published article's title, journal citation, and DOI. and (ii) their relation to the apparent emergence of unconventional criticality in such systems.

This Rapid Communication solves both of these issues by identifying the generic features of Dirac fermions interacting with the quantum fluctuations of competing symmetrybreaking order parameters using two complementary, nonperturbative approaches. For this purpose, we formulate a relativistic quantum field theory, which we analyze using advanced renormalization group (RG) methods, and which describes both the Dirac fermions and the coupled symmetrybreaking order parameters. Remarkably, we find for such systems, rather generically, a stable quantum multicritical fixed point, which moreover exhibits an emerging enhanced symmetry. Moreover, we identify an extended phase coexistence regime to expand from the high-symmetry fixed point, in which both order parameters take on finite values simultaneously. We confirm this coexistence phase also by unbiased and controlled quantum Monte Carlo (QMC) simulations. In the following, we detail these results from our combined theoretical efforts, and we also offer a natural LGW interpretation of the proposed apparent instances of fermionic DQCPs, based on our findings.

In order to arrive at such general conclusions, we consider the generic case of $(2+1)$-dimensional Dirac fermions coupled to two order parameters (OPs) with $O\left(N_{1}\right) \oplus O\left(N_{2}\right)$ symmetry, following two different and complementary routes: the nonperturbative functional RG approach (FRG) [33], and a refined QMC analysis for the model in Ref. [19]. The FRG can be performed directly in $2+1$ dimensions and allows us to establish the emergence of $O\left(N_{1}+N_{2}\right)$ symmetry at a multicritical point for all consistent values of $N_{1}, N_{2}$. Also, our approach facilitates a study of the phases with broken symmetry and we find robust indications for an 
intermediate coexistence phase for all such choices of $N_{1}, N_{2}$. Further evidence of phase coexistence for the peculiar case of $O(3) \oplus Z_{2}$ symmetry breaking is derived from our refined QMC analysis, which we adapted to accurately investigate the strong-coupling regime.

Effective quantum field theory. For a general analysis of the coupled Dirac fermions, we consider the low-energy effective Gross-Neveu-Yukawa (GNY) model with two OP fields [34-41], describing interacting spin-1/2 fermions on a honeycomb lattice in the vicinity of a multicritical point. The Euclidean Lagrangian is $\mathscr{L}=\mathscr{L}_{F}+\mathscr{L}_{B}$, where

$$
\begin{aligned}
\mathscr{L}_{F}= & \bar{\psi}\left(-i \not \partial+g_{1} M_{\phi}+g_{2} M_{\chi}\right) \psi, \\
\mathscr{L}_{B}= & \phi_{a}\left(-\partial^{2}+m_{1}^{2}\right) \phi_{a}+\chi_{b}\left(-\partial^{2}+m_{2}^{2}\right) \chi_{b} \\
& +\frac{u_{1}}{8}\left(\phi_{a} \phi_{a}\right)^{2}+\frac{u_{2}}{8}\left(\chi_{b} \chi_{b}\right)^{2}+\frac{u_{3}}{4} \phi_{a} \phi_{a} \chi_{b} \chi_{b} .
\end{aligned}
$$

The real OPs $\phi=\left(\phi_{1}, \ldots, \phi_{N_{1}}\right), \chi=\left(\chi_{1}, \ldots, \chi_{N_{2}}\right)$ act as mass terms $M_{\phi}=\gamma_{\phi}^{a} \phi_{a}$ and $M_{\chi}=\gamma_{\chi}^{b} \chi_{b}$, interacting via Yukawa couplings $\bar{g}_{1 / 2}$ with eight-component Dirac fermions $\psi$ and $\bar{\psi} \equiv \psi^{\dagger} \gamma_{0}$. Summation over repeated indices is implied. The matrices $\gamma_{\phi}^{a}, \gamma_{\chi}^{b}$ are defined as $\gamma_{\phi}^{a}=\gamma_{0} \beta_{\phi}^{a}, \gamma_{\chi}^{b}=$ $\gamma_{0} \beta_{\chi}^{b}$, where the mass matrices $\beta_{\phi}^{a}, \beta_{\chi}^{b}$ anticommute among each other. The Lagrangian $\mathscr{L}$ exhibits an effective Lorentz invariance, which is expected to emerge in two-dimensional (2D) Dirac fermion systems at criticality [26,42], and we assume this also in the vicinity of the multicritical point. The GNY model $\mathscr{L}$ requires $d+N$ anticommuting matrices, where $d$ is the spatial dimension, and $N=N_{1}+N_{2}$. For the eight-dimensional representation relevant for graphene, this implies $N \leqslant 5[43,44]$.

The $O\left(N_{1}\right) \oplus O\left(N_{2}\right)$ symmetric system in Eq. (1) includes a symmetry-enlarged $O\left(N_{1}+N_{2}\right)$-invariant subspace, when $u_{1}=u_{2}=u_{3}, m_{1}^{2}=m_{2}^{2}$, and $g_{1}^{2}=g_{2}^{2}$. The leading-order $\epsilon$ expansion $[39,40]$ studied the RG fixed point with an enlarged $O(N)$ symmetry, referred to as the isotropic fixed point (IFP). Within that approach, the IFP is found to be stable for all consistent values of $N$. However, it is well known from studies of purely bosonic $O\left(N_{1}\right) \oplus O\left(N_{2}\right)$ models [45,46] that the leading-order $\epsilon$ expansion severely overestimates the stability of the IFP. In contrast, FRG provides more faithful results already at truncation orders that we exploit here [47].

An important subtlety in Dirac fermion systems concerns the determination of the nature of a multicritical point. In a purely bosonic theory, a multicritical point can either be bicritical or tetracritical, and the two cases can be distinguished by the sign of the quantity $\Delta=u_{1} u_{2}-u_{3}^{2}$ in terms of the quartic couplings $u_{i}$ at the RG fixed point, which is bicritical if $\Delta<0$ and tetracritical if $\Delta>0[45,47,48]$. For the symmetry-enhanced case, $\Delta=0$, the above classification is valid if the submanifold in coupling space determined by $\Delta=0$ is closed under the RG flow. This is not generally the case in theories with massless fermions as can be shown using the $\epsilon$ expansion [39]: The submanifold in theory space defined by $u_{1}=u_{2}=u_{3}=: u \neq 0$ satisfies $\Delta=0$, but its dependence on the logarithmic RG scale $t$ is given by $\partial_{t} \Delta=2 u\left(g_{1}^{2}-g_{2}^{2}\right)^{2}$. Therefore, in the presence of Dirac fermions, the sign of $\Delta$ may be subject to change.

Functional renormalization group. We employ the nonperturbative FRG approach $[33,49]$ to evaluate the generating functional of one-particle irreducible $n$-point correlation functions $\Gamma^{(n)}$; see Refs. [50-60] for applications to lowdimensional GNY systems. This method allows us to calculate directly in $D=2+1$ and integrate out the flow also within the ordered phases. Central to the FRG method is the exact RG flow equation $\partial_{k} \Gamma_{k}=\frac{1}{2} \operatorname{Tr}\left[\partial_{k} R_{k}\left(\Gamma_{k}^{(2)}+R_{k}\right)^{-1}\right]$ for the average effective action $\Gamma_{k}$, where $\Gamma_{k}^{(2)}$ is the second functional derivative with respect to all field degrees of freedom, and $R_{k}$ is an infrared cutoff function. This flow equation interpolates between the bare action at the ultraviolet (UV) cutoff $k=\Lambda$ and the full quantum effective action $\Gamma=\Gamma_{k=0}$.

We employ a truncation based on the original form of the microscopic action in Eq. (1), i.e.,

$$
\begin{aligned}
\Gamma_{k}= & \int d^{D} x\left\{\bar{\psi}\left(-i Z_{\psi, k} \not \partial+g_{1, k} M_{\phi}+g_{2, k} M_{\chi}\right) \psi\right. \\
& \left.-\frac{1}{2} Z_{\phi, k} \phi_{a} \partial^{2} \phi_{a}-\frac{1}{2} Z_{\chi, k} \chi_{b} \partial^{2} \chi_{b}+V_{k}\left(\rho_{\phi}, \rho_{\chi}\right)\right\},
\end{aligned}
$$

which is known as the extended local potential approximation, LPA'. The scale dependence of the Yukawa couplings $g_{1, k}$ and $g_{2, k}$ as well as the wave-function renormalizations $Z_{\Phi, k}$ have been made explicit. The bosonic potential $V_{k}$ depends on the $O\left(N_{1}\right)$ and $O\left(N_{2}\right)$ invariant quantities $\rho_{\phi}=\phi_{a} \phi_{a} / 2, \rho_{\chi}=$ $\chi_{b} \chi_{b} / 2$, and we expand its dimensionless form $u=k^{-D} V_{k}$ as a function of the dimensionless $O\left(N_{i}\right)$-symmetric field variables $\tilde{\rho}_{\phi}=Z_{\phi, k} k^{2-D} \rho_{\phi}$ and $\tilde{\rho}_{\chi}=Z_{\chi, k} k^{2-D} \rho_{\chi}$ around a (running) minimum, denoted $\kappa_{\phi / \chi}=\tilde{\rho}_{\phi / \chi}$,min. A truncation up to order $n_{\max }$ in the fields will be referred to in the following as $\mathrm{LPA}^{\prime} n_{\text {max }}$. The nature of the minimum corresponds to three different scenarios, depending on whether the individual OP fields are in the symmetric or in the spontaneously symmetrybroken regime: (1) Both OP fields remain in their symmetric phases, i.e., the minimum of the potential is at $\kappa_{\phi / \chi}=0$. (2) The OP $\phi$ acquires an expectation value, while the other OP $\chi$ remains in its symmetric phase-similarly, for the reversed situation. (3) The minimum of the potential is at nonzero expectation values for both OPs, i.e., $\kappa_{\phi / \chi} \neq 0$. Note that our ansatz for the effective action does not include topological terms, which naturally occur only when the fermions are massive and can be integrated out [61].

The FRG flow equations for the dimensionless expansion coefficients of the potential $u$, including the quadratic mass terms and the quartic couplings, the dimensionless Yukawa couplings $\tilde{g}_{i, k}^{2}=g_{i}^{2} k^{D-4} Z_{i, k}^{-1} Z_{\psi, k}^{-2}$, as well as the anomalous dimensions $\partial_{t} Z_{\psi / \phi / \chi}$ are obtained by taking the corresponding derivatives of the ansatz in Eq. (2). Flow equations for the expectation values $\kappa_{\phi / \chi}$ are obtained from the condition that they are a minimum (cf. the Supplemental Material [62] for details). This procedure yields a set of coupled flow equations of the form $\partial_{t} x_{i}=\beta_{i}\left(\left\{x_{j}\right\}\right)$ in the space of coupling parameters $x_{j}$.

Stability of the symmetry-enhanced fixed point. A fixed point is defined by the condition $\forall i: \beta_{i}\left(\left\{x_{j}\right\}\right)=0$. Here, we focus on the IFP, facilitating the symmetry enhancement $O\left(N_{1}\right) \oplus O\left(N_{2}\right) \rightarrow O(N)$. The system has two tuning parameters represented by the boson masses $m_{i}^{2}$ [cf. Eq. (1)]. They give rise to two relevant directions in the RG flow with scaling properties given by the critical exponents $\theta_{1}$ and $\theta_{2}$ obtained 
TABLE I. Largest five eigenvalues $\theta_{i}$ of the stability matrix based on FRG calculation in LPA'8 truncation, for different values of $N_{1}+$ $N_{2}$. Eigenvalues that already appear in the $O\left(N_{1}+N_{2}\right)$ symmetric models are printed in boldface.

\begin{tabular}{lccccc}
\hline \hline$N_{1}+N_{2}$ & $\theta_{1}$ & $\theta_{2}$ & $\theta_{3}$ & $\theta_{4}$ & $\theta_{5}$ \\
\hline 2 & 0.878 & $\mathbf{0 . 8 6 4}$ & $\mathbf{- 0 . 8 7 8}$ & -1.087 & -1.109 \\
3 & $\mathbf{0 . 7 7 3}$ & 0.726 & $\mathbf{- 0 . 9 2 4}$ & -1.179 & -1.322 \\
4 & $\mathbf{0 . 7 3 4}$ & 0.580 & $\mathbf{- 1 . 0 1 7}$ & -1.274 & -1.542 \\
5 & $\mathbf{0 . 7 3 8}$ & 0.465 & $\mathbf{- 1 . 1 3 2}$ & -1.361 & -1.732 \\
\hline \hline
\end{tabular}

from diagonalizing the stability matrix $\mathcal{M}_{i j}:=-\left.\left(\partial \beta_{i} / \partial x_{j}\right)\right|_{x^{*}}$ (here, $x_{j}^{*}$ denote the fixed-point coordinates). Positive eigenvalues $\theta_{i}$ of $\mathcal{M}_{i j}$ correspond to relevant directions, i.e., their number indicates the number of tuning parameters. Negative $\theta_{i}$ correspond to irrelevant directions, attracted to the fixed point. The IFP is stable, if the third-largest eigenvalue of the $\mathcal{M}_{i j}$ is negative (called the stability exponent).

We report the numerical results for the five largest eigenvalues of the stability matrix in Table I, exhibiting the stability of the IFP for all consistent choices of $N$. In particular, the stability exponent has a sizable magnitude of $\mathcal{O}(1)$. Therefore, any perturbation of the enhanced symmetry near the multicritical point will die out rather quickly, supporting a strong tendency towards the emergent symmetry. This strong tendency towards emergent $O\left(N_{1}+N_{2}\right)$ is consistent with the QMC findings in Ref. [19]. Noticeable, the symmetry enhancement is naturally realized in our extended LGW approach through the fluctuations of massless Dirac fermions, i.e., without requiring the inclusion of additional topological terms. This contrasts to the case of purely bosonic $O\left(N_{1}\right) \oplus O\left(N_{2}\right)$ models [45,47], for which symmetry enhancement is supported only for $N_{1}=$ $N_{2}=1$, with an almost marginal stability exponent.

Phase diagram from field theory. The phase diagram finally can be obtained from integrating the FRG flow towards the infrared. To this end, we formulate the initial value problem at an arbitrary ultraviolet scale $\Lambda$, used to set the units. To resolve the phase diagram in the vicinity of the symmetryenhanced quantum multicritical point, we perform a sweep of initial conditions in the vicinity of the IFP. We consider three different choices for $N_{1}$ and $N_{2}$ : (i) two coupled Ising order parameters $\left(N_{1}=N_{2}=1\right)$, (ii) a $O(3) \oplus \mathbb{Z}_{2}$ model $\left(N_{1}=3\right.$, $N_{2}=1$ ), relevant to the model in Ref. [19], and (iii) a $O(3) \oplus$ $O(2)$ model $\left(N_{1}=3, N_{2}=2\right)$. The resulting phase diagrams are shown in Fig. 1, and we find that all cases exhibit extended coexistence regions, at which both OPs develop a vacuum expectation value.

In the region close to the line $\delta m_{1}^{2}=\delta m_{2}^{2}$ in Fig. 1 emerging from the IFP, Goldstone fluctuations in both adjacent phases appear when the spontaneously broken symmetry is continuous. Their interplay with the massive modes has the tendency to drive the system out of the symmetry-broken phase. Indeed, when the adjacent symmetry-broken phases involve no massless modes (for $N_{1}=N_{2}=1$ ), the line of exact $O(N)$ symmetry can be resolved effortlessly. In the presence of Goldstone modes and very close to $\delta m_{1}^{2}=\delta m_{2}^{2}$, however, the expectation values $\kappa_{i, k}$ do not straightforwardly converge to definite values in the limit $k \rightarrow 0$, within our
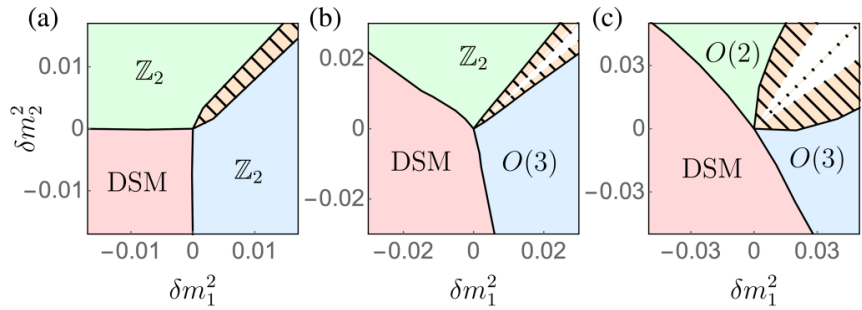

FIG. 1. Phase diagrams from FRG for $N_{1}=1, N_{2}=1$ (left), $N_{1}=3, N_{2}=1$ (middle), and $N_{1}=3, N_{2}=2$ (right). Tuning parameters $\delta m_{i}^{2}$ measure the distance from the IFP, DSM denotes the Dirac semimetal regime, and other phases are labeled by the broken symmetry. Solid lines are continuous transitions, and coexistence regions are shown in shaded orange. Within the white areas, the FRG flow is numerically unstable.

approximation of the exact RG flow (see Ref. [62] for a more detailed discussion). Nevertheless, sufficiently away from that parameter regime, we can reliably establish the appearance of a stable coexistence region near the IFP. Moreover, exactly along the line of enhanced symmetry $\left(\delta m_{1}^{2}=\delta m_{2}^{2}\right)$, we can integrate out the renormalization group flow by exploiting the enhanced symmetry, and find that a coexistence of both orders occurs. The missing regions of the phase diagrams may be accessible to alternative FRG methods, e.g., by pseudospectral methods [56,57].

Quantum Monte Carlo. For the case of $O(3) \oplus \mathbb{Z}_{2}$, we obtain direct support for the coexistence region also from a refined QMC analysis for the microscopic model of Ref. [19]. For this purpose, we derive an effective quantum spin model that emerges in the strongly interacting regime of the interacting Dirac fermion model, and which can be simulated by more efficient, sign problem-free QMC methods using cluster updates and larger lattices than accessible to the QMC approach for the original fermionic model. The Hamiltonian of this effective quantum spin model, obtained by perturbation theory about the strong-interaction limit [63], reads

$$
\begin{aligned}
H= & \sum_{\langle i, j\rangle}\left(J_{i j}+\chi_{i j} \sigma_{\langle i j\rangle}^{z}\right)\left(\mathbf{S}_{i} \cdot \mathbf{S}_{j}-\frac{1}{4}\right)-J_{I} \sum_{\langle i j, k l\rangle} \sigma_{\langle i j\rangle}^{z} \sigma_{\langle k l\rangle}^{z} \\
& -h \sum_{\langle i, j\rangle} \sigma_{\langle i j\rangle}^{x},
\end{aligned}
$$

in terms of Heisenberg $S=1 / 2$ spins $\mathbf{S}_{i}$, residing on a honeycomb lattice and coupled via bond-centered strengths $\chi_{i j}$ to a transverse-field $(h)$ Ising model of spins $\sigma_{\langle i j\rangle}$, located on the nearest-neighbor bonds of the honeycomb lattice. The summation over $\langle i, j\rangle(\langle i j, k l\rangle)$ extends over nearestneighbor Heisenberg (Ising model) spins. In terms of the nearest-neighbor hopping $t$, the Hubbard interaction $U$, and the fermion-spin couplings $\xi_{i j}$ of the underlying fermionic model, we obtain $J_{i j}=4\left(t^{2}+\xi_{i j}^{2}\right) / U$, and $\chi_{i j}=8 t \xi_{i j} / U$ in second-order perturbation theory [62]. These relations can also be used to specify parameter values of $H$ and compare to the results of Ref. [19].

With a staggered pattern of $\xi_{i j}=+\xi, 0,-\xi$ (cf. the inset of Fig. 2) as in Ref. [19], the model has a combined lattice inversion and Ising model spin-flip $\mathbb{Z}_{2}$ symmetry in addition to the $O(3)$ [SU(2)] symmetry of the Heisenberg 


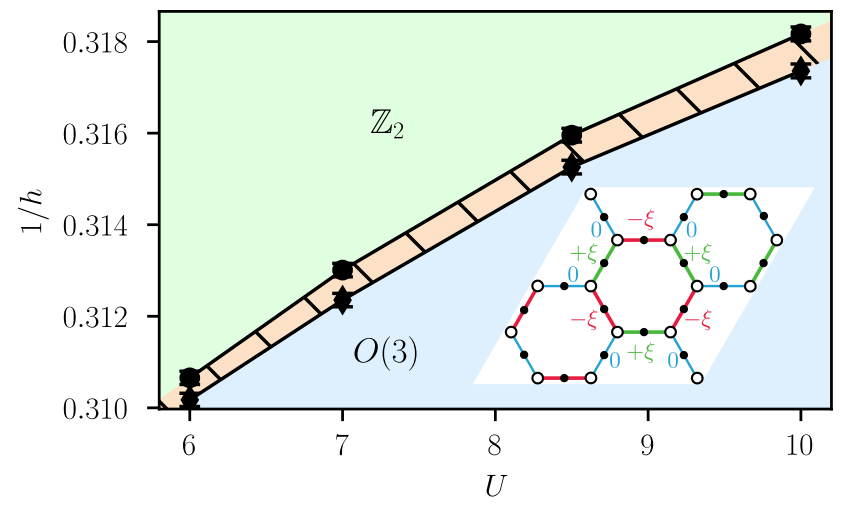

FIG. 2. QMC phase diagram of the $O(3) \oplus \mathbb{Z}_{2}$ model $H$ in terms of the parameters $(U, 1 / h)$ of the underlying fermionic model $\left(\xi=0.5, J_{I}=1, t=1\right)$. The coexistence region is shown in shaded orange. The inset shows the honeycomb lattice with the modulated pattern $\xi_{i j}=-\xi, 0,+\xi$ in different colors. Open (solid) circles denote Heisenberg (Ising) spins.

exchange. To connect to the previous results, we also fix $\xi=0.5, J_{I}=1$, and $t=1$, and probe the strong-coupling regime for values of $U>6$, where large single-particle gaps prevailed. We used a hybrid QMC parallel tempering scheme $[62,64,65]$ for the Hamiltonian $H$ on periodic lattices with $N_{\mathrm{H}}$ Heisenberg spins (and $N_{\mathrm{I}}=3 N_{\mathrm{H}} / 2$ Ising spins), for $N_{\mathrm{H}}$ up to 2400 , based on the stochastic series expansion approach [66-68]. In particular, we monitored the evolution of the ferromagnetic Ising OP $m_{\mathrm{I}}=\left\langle\left|\frac{1}{N_{\mathrm{L}}} \sum_{b=1}^{N_{\mathrm{I}}} \sigma_{b}^{z}\right|\right\rangle$ and the antiferromagnetic Heisenberg OP $m_{\mathrm{H}}=\left\langle\left|\frac{1}{N_{\mathrm{H}}} \sum_{i=1}^{N_{\mathrm{H}}}(-1)^{i} S_{i}^{z}\right|\right\rangle$ upon varying $h$. As an example, Fig. 3 shows the finite-size scaling of both OPs, for $h$ in the transition region at $U=7$. The algebraic behaviors at the order-to-disorder transitions are in accord with the anticipated asymptotic scalings for the purely bosonic Ising and Heisenberg universality classes in dimension $D=2+1$, and yield two distinct critical field strengths,

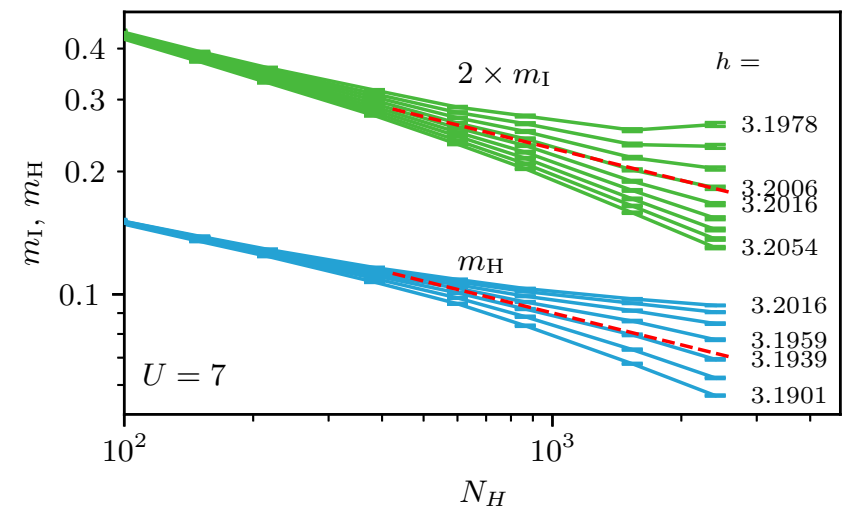

FIG. 3. Finite-size scaling of $m_{\mathrm{H}}$ and $m_{\mathrm{I}}$ for $h$ in the transition region of $H$ for $U=7\left(\xi=0.5, J_{I}=1, t=1\right)$. Dashed lines show the asymptotic scalings $m_{j} \propto N_{j}^{-\beta_{j} / 2 v_{j}}$ for $j=\mathrm{I}, \mathrm{H}$, with $\beta_{j}, v_{j}$ as of Ref. [69]. For clarity, the values for $m_{\mathrm{I}}$ were multiplied by a factor of 2 . The lowest and highest shown values of $h$ are given, as well as the near-critical $h$ values.
$h_{c}^{I}=3.200(1)$ for $m_{\mathrm{I}}$, and $h_{c}^{H}=3.194(1)$ for $m_{\mathrm{H}}$. These values are consistent also with an analysis of the corresponding Binder ratios [62].

The resulting phase diagram in the above parameter regime is shown in Fig. 2. It exhibits a narrow intermediate phase of coexisting orders between the small (large) $h$ phase with pure $\mathbb{Z}_{2}[O(3)]$ symmetry breaking: For small $h$, the Ising model spins order ferromagnetically, spontaneously breaking the $\mathbb{Z}_{2}$ symmetry. Due to the staggered $\xi_{i j}$ coupling, this induces a preferred dimerization pattern on the honeycomb lattice, which leads to a dominant valence-bond singlet formation of the Heisenberg spins along the stronger $\left(\xi_{i j} \sigma_{\langle i j\rangle}^{z}>0\right)$ bonds. Upon increasing $h$, the ferromagnetic order reduces, in effect weakening also the induced dimerization, so that antiferromagnetic Heisenberg spin order can eventually set in prior to the full suppression of the ferromagnetic Ising spin order at even larger values of $h$. Due to the large single-particle gaps for $U>6$, we exclude residual (charge) fluctuations in the fermionic model to qualitatively modify this basic physics. We observe in Fig. 2 that the coexistence regime widens slightly with increasing $U$. This thinness of the coexistence regime within the considered parameter regime explains why it was not resolved by the fermionic QMC methods [19]. It should, however, be noted that within the effective quantum spin model we cannot identify the multicritical point of the underlying fermionic theory, which was located at $(U, 1 / h) \approx$ $(4.2,0.28)$ in Ref. [19]. Indeed, the multicritical IFP is of genuine fermionic nature (cf. the FRG results).

Discussion. We studied $(2+1)$-dimensional Dirac fermions coupled to two compatible OPs with $O\left(N_{1}\right)$ and $O\left(N_{2}\right)$ symmetry in the vicinity of the multicritical isotropic fixed point, providing an emergent $O\left(N_{1}+N_{2}\right)$ symmetry. Our FRG study predicts a strong irrelevance of perturbations of the $O\left(N_{1}+N_{2}\right)$ symmetry, which is consistent with the numerical result of an emergent symmetry in the related Dirac fermion lattice model [19]. Contrary to this numerical study, however, we do not find a single line of direct, continuous order-to-order transitions. Instead, we identify a robust region of coexistence of both orders, which is separated by continuous transitions from the other phases. In the case of the $O(3) \oplus \mathbb{Z}_{2}$ symmetry, relevant for Ref. [19], we could furthermore support this field-theoretical analysis by large-scale QMC simulations. Therefore, our combined approach sheds light on the nature of quantum critical fermions coupled to bosonic fields as in Ref. [19], and further challenges the scenario of deconfined quantum criticality in quantum spin-Hall insulators [18]. It appears promising to also experimentally probe the physics that we uncovered here, given the very recent advances in the experimental studies of twisted bilayer graphene with Dirac points indeed located in proximity to several competing orders [70].

Acknowledgments. We are grateful to F. F. Assaad and I. F. Herbut for discussions. E.T. was supported by the Deutsche Forschungsgemeinschaft (DFG) through the Leibniz Prize of A. Rosch, RO2265/5-1. L.J. was supported by the DFG through JA2306/4-1 (Emmy Noether program, project id 411750675), SFB 1143 (project id 247310070), and the Würzburg-Dresden Cluster of Excellence ct.qmat (EXC 2147, project id 390858490). M.M.S. was supported by the DFG, 
project id 277146847 - SFB 1238 (C02, C03). L.W. and S.W. acknowledge support by DFG under Grant No. WE/3649/4-2 of the FOR 1807 and through RTG 1995. Fur- thermore, we acknowledge the IT Center at RWTH Aachen University and the JSC Jülich for access to computing time through JARA-HPC.
[1] Y. Cao, V. Fatemi, A. Demir, S. Fang, S. L. Tomarken, J. Y. Luo, J. D. Sanchez-Yamagishi, K. Watanabe, T. Taniguchi, E. Kaxiras et al., Correlated insulator behaviour at half-filling in magic-angle graphene superlattices, Nature (London) 556, 80 (2018).

[2] Y. Cao, V. Fatemi, S. Fang, K. Watanabe, T. Taniguchi, E. Kaxiras, and P. Jarillo-Herrero, Unconventional superconductivity in magic-angle graphene superlattices, Nature (London) 556, 43 (2018).

[3] P. A. Lee, N. Nagaosa, and X.-G. Wen, Doping a Mott insulator: Physics of high-temperature superconductivity, Rev. Mod. Phys. 78, 17 (2006).

[4] B. Keimer, S. A. Kivelson, M. R. Norman, S. Uchida, and J. Zaanen, From quantum matter to high-temperature superconductivity in copper oxides, Nature (London) 518, 179 (2015).

[5] A. Kerelsky, L. J. McGilly, D. M. Kennes, L. Xian, M. Yankowitz, S. Chen, K. Watanabe, T. Taniguchi, J. Hone, C. Dean et al., Maximized electron interactions at the magic angle in twisted bilayer graphene, Nature (London) 572, 95 (2019).

[6] Y. Choi, J. Kemmer, Y. Peng, A. Thomson, H. Arora, R. Polski, Y. Zhang, H. Ren, J. Alicea, G. Refael et al., Electronic correlations in twisted bilayer graphene near the magic angle, Nat. Phys. 15, 1174 (2019).

[7] M. Yankowitz, S. Chen, H. Polshyn, Y. Zhang, K. Watanabe, T. Taniguchi, D. Graf, A. F. Young, and C. R. Dean, Tuning superconductivity in twisted bilayer graphene, Science 363, 1059 (2019).

[8] X. Lu, P. Stepanov, W. Yang, M. Xie, M. A. Aamir, I. Das, C. Urgell, K. Watanabe, T. Taniguchi, G. Zhang et al., Superconductors, orbital magnets, and correlated states in magic angle bilayer graphene, Nature (London) 574, 653 (2019).

[9] Y. Da Liao, Z. Y. Meng, and X. Y. Xu, Valence Bond Orders at Charge Neutrality in a Possible Two-Orbital Extended Hubbard Model for Twisted Bilayer Graphene, Phys. Rev. Lett. 123, 157601 (2019).

[10] H.-K. Tang, J. N. Leaw, J. N. B. Rodrigues, I. F. Herbut, P. Sengupta, F. F. Assaad, and S. Adam, The role of electronelectron interactions in two-dimensional Dirac fermions, Science 361, 570 (2018).

[11] S. Hesselmann, T. C. Lang, M. Schuler, S. Wessel, and A. M. Läuchli, Comment on "The role of electron-electron interactions in two-dimensional Dirac fermions", Science 366, eaav6869 (2019).

[12] C. Chen, X. Y. Xu, Z. Y. Meng, and M. Hohenadler, Charge-Density-Wave Transitions of Dirac Fermions Coupled to Phonons, Phys. Rev. Lett. 122, 077601 (2019).

[13] T. C. Lang and A. M. Läuchli, Quantum Monte Carlo Simulation of the Chiral Heisenberg Gross-Neveu-Yukawa Phase Transition with a Single Dirac Cone, Phys. Rev. Lett. 123, 137602 (2019).

[14] L. Iliesiu, F. Kos, D. Poland, S. S. Pufu, and D. SimmonsDuffin, Bootstrapping 3D fermions with global symmetries, J. High Energy Phys. 01 (2018) 036.
[15] M. Schuler, S. Hesselmann, S. Whitsitt, T. C. Lang, S. Wessel, and A. M. Läuchli, Torus spectroscopy of the Gross-NeveuYukawa quantum field theory: Free Dirac versus chiral Ising fixed point, arXiv:1907.05373.

[16] L. D. Landau and E. M. Lifshitz, Statistical Physics, Part 1, Course of Theoretical Physics Vol. 5 (Butterworth-Heinemann, Oxford, UK, 1980).

[17] T. Senthil, L. Balents, S. Sachdev, A. Vishwanath, and M. P. A Fisher, Quantum criticality beyond the Landau-GinzburgWilson paradigm, Phys. Rev. B 70, 144407 (2004).

[18] Y. Liu, Z. Wang, T. Sato, M. Hohenadler, C. Wang, W. Guo, and F. F. Assaad, Superconductivity from the condensation of topological defects in a quantum spin-Hall insulator, Nat. Commun. 10, 2658 (2019).

[19] T. Sato, M. Hohenadler, and F. F. Assaad, Dirac Fermions with Competing Orders: Non-Landau Transition with Emergent Symmetry, Phys. Rev. Lett. 119, 197203 (2017).

[20] P. Serna and A. Nahum, Emergence and spontaneous breaking of approximate $O(4)$ symmetry at a weakly first-order deconfined phase transition, Phys. Rev. B 99, 195110 (2019).

[21] Y. Q. Qin, Y.-Y. He, Y.-Z. You, Z.-Y. Lu, A. Sen, A. W. Sandvik, C. Xu, and Z. Y. Meng, Duality between the Deconfined Quantum-Critical Point and the Bosonic Topological Transition, Phys. Rev. X 7, 031052 (2017).

[22] S. Gazit, F. F. Assaad, S. Sachdev, A. Vishwanath, and C. Wang, Confinement transition of $\mathbb{Z}_{2}$ gauge theories coupled to massless fermions: Emergent quantum chromodynamics and SO(5) symmetry, Proc. Natl. Acad. Sci. USA 115, E6987 (2018).

[23] B. Roy, Multicritical behavior of $\mathbb{Z}_{2} \times O(2)$ Gross-NeveuYukawa theory in graphene, Phys. Rev. B 84, 113404 (2011).

[24] S.-C. Zhang, A unified theory based on $S O(5)$ symmetry of superconductivity and antiferromagnetism, Science 275, 1089 (1997).

[25] S.-K. Jian, Y.-F. Jiang, and H. Yao, Emergent Spacetime Supersymmetry in 3D Weyl Semimetals and 2D Dirac Semimetals, Phys. Rev. Lett. 114, 237001 (2015).

[26] B. Roy, V. Juricic, and I. F. Herbut, Emergent Lorentz symmetry near fermionic quantum critical points in two and three dimensions, J. High Energy Phys. 04 (2016) 018.

[27] Z.-X. Li, Y.-F. Jiang, S.-K. Jian, and H. Yao, Fermion-induced quantum critical points, Nat. Commun. 8, 314 (2017).

[28] S.-K. Jian and H. Yao, Fermion-induced quantum critical points in two-dimensional Dirac semimetals, Phys. Rev. B 96, 195162 (2017).

[29] S.-K. Jian and H. Yao, Fermion-induced quantum critical points in three-dimensional Weyl semimetals, Phys. Rev. B 96, 155112 (2017).

[30] M. M. Scherer and I. F. Herbut, Gauge-field-assisted Kekulé quantum criticality, Phys. Rev. B 94, 205136 (2016).

[31] L. Classen, I. F. Herbut, and M. M. Scherer, Fluctuationinduced continuous transition and quantum criticality in Dirac semimetals, Phys. Rev. B 96, 115132 (2017). 
[32] E. Torres, L. Classen, I. F. Herbut, and M. M. Scherer, Fermioninduced quantum criticality with two length scales in Dirac systems, Phys. Rev. B 97, 125137 (2018).

[33] C. Wetterich, Exact evolution equation for the effective potential, Phys. Lett. B 301, 90 (1993).

[34] B. Roy and V. Juričić, Strain-induced time-reversal odd superconductivity in graphene, Phys. Rev. B 90, 041413(R) (2014).

[35] L. Classen, I. F. Herbut, L. Janssen, and M. M. Scherer, Mott multicriticality of Dirac electrons in graphene, Phys. Rev. B 92, 035429 (2015).

[36] I. F. Herbut, Interactions and Phase Transitions on Graphene's Honeycomb Lattice, Phys. Rev. Lett. 97, 146401 (2006).

[37] I. F. Herbut, V. Juričić, and B. Roy, Theory of interacting electrons on the honeycomb lattice, Phys. Rev. B 79, 085116 (2009).

[38] L. Classen, I. F. Herbut, L. Janssen, and M. M. Scherer, Competition of density waves and quantum multicritical behavior in Dirac materials from functional renormalization, Phys. Rev. B 93, 125119 (2016).

[39] L. Janssen, I. F. Herbut, and M. M. Scherer, Compatible orders and fermion-induced emergent symmetry in Dirac systems, Phys. Rev. B 97, 041117(R) (2018).

[40] B. Roy, P. Goswami, and V. Juričić, Itinerant quantum multicriticality of two-dimensional Dirac fermions, Phys. Rev. B 97, 205117 (2018).

[41] B. Roy and V. Juričić, Fermionic multicriticality near Kekulé valence-bond ordering on a honeycomb lattice, Phys. Rev. B 99, 241103(R) (2019).

[42] S. Ray, M. Vojta, and L. Janssen, Quantum critical behavior of two-dimensional Fermi systems with quadratic band touching, Phys. Rev. B 98, 245128 (2018).

[43] S. Ryu, C. Mudry, C.-Y. Hou, and C. Chamon, Masses in graphenelike two-dimensional electronic systems: Topological defects in order parameters and their fractional exchange statistics, Phys. Rev. B 80, 205319 (2009).

[44] I. F. Herbut, Isospin of topological defects in Dirac systems, Phys. Rev. B 85, 085304 (2012).

[45] P. Calabrese, A. Pelissetto, and E. Vicari, Multicritical phenomena in $\mathrm{O}\left(n_{1}\right) \bigoplus \mathrm{O}\left(n_{2}\right)$-symmetric theories, Phys. Rev. B 67, 054505 (2003).

[46] E. Vicari, Critical phenomena and renormalization-group flow of multi-parameter $\Phi^{4}$ field theories, Proceedings of the 25th International Symposium on Lattice Field Theory (LATTICE 2007), PoS 42, 023 (2007).

[47] A. Eichhorn, D. Mesterházy, and M. M. Scherer, Multicritical behavior in models with two competing order parameters, Phys. Rev. E 88, 042141 (2013).

[48] K.-S. Liu and M. E. Fisher, Quantum lattice gas and the existence of a supersolid, J. Low Temp. Phys. 10, 655 (1973).

[49] J. Berges, N. Tetradis, and C. Wetterich, Nonperturbative renormalization flow in quantum field theory and statistical physics, Phys. Rep. 363, 223 (2002).

[50] L. Rosa, P. Vitale, and C. Wetterich, Critical Exponents of the Gross-Neveu Model from the Effective Average Action, Phys. Rev. Lett. 86, 958 (2001).

[51] F. Hofling, C. Nowak, and C. Wetterich, Phase transition and critical behavior of the $d=3$ Gross-Neveu model, Phys. Rev. B 66, 205111 (2002).
[52] H. Gies, L. Janssen, S. Rechenberger, and M. M. Scherer, Phase transition and critical behavior of $d=3$ chiral fermion models with left/right asymmetry, Phys. Rev. D 81, 025009 (2010).

[53] L. Janssen and H. Gies, Critical behavior of the $(2+1)$ dimensional Thirring model, Phys. Rev. D 86, 105007 (2012).

[54] J. Braun, H. Gies, and D. D. Scherer, Asymptotic safety: A simple example, Phys. Rev. D 83, 085012 (2011).

[55] D. D. Scherer, J. Braun, and H. Gies, Many-flavor phase diagram of the (2+1)D Gross-Neveu model at finite temperature, J. Phys. A: Math. Theor. 46, 285002 (2013).

[56] B. Knorr, Ising and Gross-Neveu model in next-to-leading order, Phys. Rev. B 94, 245102 (2016).

[57] B. Knorr, Critical chiral Heisenberg model with the functional renormalization group, Phys. Rev. B 97, 075129 (2018).

[58] L. Janssen and I. F. Herbut, Antiferromagnetic critical point on graphene's honeycomb lattice: A functional renormalization group approach, Phys. Rev. B 89, 205403 (2014).

[59] G. P. Vacca and L. Zambelli, Multimeson Yukawa interactions at criticality, Phys. Rev. D 91, 125003 (2015).

[60] P. Feldmann, A. Wipf, and L. Zambelli, Critical Wess-Zumino models with four supercharges in the functional renormalization group approach, Phys. Rev. D 98, 096005 (2018).

[61] A. G. Abanov and P. B. Wiegmann, Theta terms in nonlinear sigma models, Nucl. Phys. B 570, 685 (2000).

[62] See Supplemental Material at http://link.aps.org/supplemental/ 10.1103/PhysRevResearch.2.022005 for details on the FRG calculations, the derivation of the effective quantum spin model, and the hybrid QMC scheme.

[63] A. H. MacDonald, S. M. Girvin, and D. Yoshioka, $\frac{t}{U}$ expansion for the Hubbard model, Phys. Rev. B 37, 9753 (1988).

[64] K. Hukushima, H. Takayama, and K. Nemoto, Application of an extended ensemble method to spin glasses, Int. J. Mod. Phys. C 07, 337 (1996).

[65] K. Hukushima and K. Nemoto, Exchange Monte Carlo method and application to spin glass simulations, J. Phys. Soc. Jpn. 65, 1604 (1996).

[66] A. W. Sandvik, Stochastic series expansion method with operator-loop update, Phys. Rev. B 59, R14157(R) (1999).

[67] A. W. Sandvik, Stochastic series expansion method for quantum Ising models with arbitrary interactions, Phys. Rev. E 68, 056701 (2003).

[68] P. Sengupta, A. W. Sandvik, and D. K. Campbell, Bondorder-wave phase and quantum phase transitions in the onedimensional extended Hubbard model, Phys. Rev. B 65, 155113 (2002)

[69] F. Kos, D. Poland, D. Simmons-Duffin, and A. Vichi, Precision islands in the Ising and $O(N)$ models, J. High Energy Phys. 08 (2016) 036.

[70] U. Zondiner, A. Rozen, D. Rodan-Legrain, Y. Cao, R. Queiroz, T. Taniguchi, K. Watanabe, Y. Oreg, F. von Oppen, A. Stern, E. Berg, P. Jarillo-Herrero, and S. Ilani, Cascade of phase transitions and Dirac revivals in magic angle graphene, arXiv:1912.06150.

Correction: The unnumbered equation in the "Quantum Monte Carlo" section contained an omission that had consequences for Figs. 2 and 3, the caption to Fig. 3, and the values for $h_{c}^{I}$ and $h_{c}^{H}$; all have been fixed. 